\title{
The fine-tuning price of the early LHC
}

\author{
Alessandro Strumia \\ Dipartimento di Fisica dell'Università di Pisa and INFN, Italia \\ National Institute of Chemical Physics and Biophysics, Ravala 10, Tallin, Estonia
}

\begin{abstract}
LHC already probed and excluded most of the parameter space of the Constrained Minimal Supersymmetric Standard Model allowed by previous experiments. Only about $0.3 \%$ of the CMSSM parameter space survives. This fraction rises to about $0.9 \%$ if the bound on the Higgs mass can be circumvented.
\end{abstract}

\section{Introduction}

When LEP started 20 years ago, the main topic of high-energy physics was finding the right supersymmetric unified model, its embedding in string theory and understanding how it predicts a zero cosmological constant.

But supersymmetry was not found and LEP opened a "little hierarchy problem" [1, 2]: experimental bounds on sparticle masses made difficult to fully solve the higgs mass hierarchy problem. Furthermore, cosmological observations strongly suggested a small but non-zero cosmological constant [3], opening a new hierarchy problem with no known solution.

Now LHC is starting and its most fundamental goal is telling why the the weak scale is much below the Planck scale: is it small due to some natural reason (as many theorists expect) or for some other "unnatural" reason (such as anthropic selection in a multiverse)?

Supersymmetry remains the main candidate natural solution to the weak scale hierarchy problem. The CMS and ATLAS collaborations published the first results of searches for supersymmetric particles looking at events with jets and missing energy, without and with a lepton, first in data at $\sqrt{s}=7 \mathrm{TeV}$ with $35 / \mathrm{pb}$ of integrated luminosity [4], later increased to about $1.1 / \mathrm{fb}[5]$.

Supersymmetry was not found in such LHC data, and the LHC collaborations produced bounds in the CMSSM model: degenerate squarks and gluinos must be typically heavier than up to $1.1 \mathrm{TeV}$ (see fig. 2 below). This is significantly stronger than previous bounds.

Again, the main implications of such negative experimental searches concerns naturalness, which is the heart of the main question: is the weak scale naturally small? 


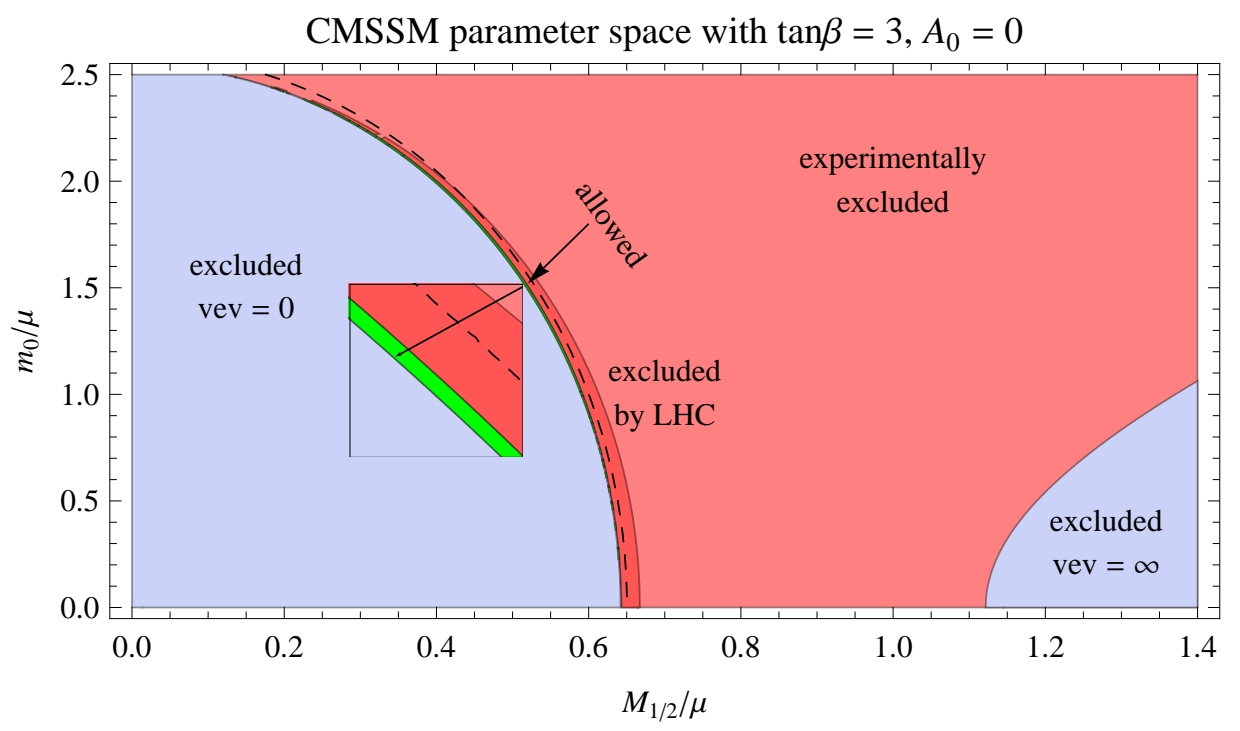

Figure 1: A typical example of the parameter space of the CMSSM model. The green region is allowed (see it in the enlarged box). The dashed line around the boundary of the allowed region is the prediction of the model considered in [13].

\section{Naturalness}

To illustrate the naturalness problem of the CMSSM model we recall that it predicts the $Z$ mass to be

$$
M_{Z}^{2} \approx 0.7 M_{3}^{2}+0.2 m_{0}^{2}-2 \mu^{2}=(91 \mathrm{GeV})^{2} \times 100\left(\frac{M_{3}}{1.1 \mathrm{TeV}}\right)^{2}+\cdots
$$

where $M_{3} \approx 2.6 M_{1 / 2}$ is the gluino mass, $M_{1 / 2}$ and $m_{0}$ are the unified gaugino and scalar masses at the unification scale; the $\mu$ term is renormalized at the weak scale, and $\cdots$ denotes the $m_{0}^{2}$ and $\mu^{2}$ terms. We here assumed $\tan \beta=3$ and $A_{0}=0$, such that the top Yukawa coupling renormalized at the unification scale is $\lambda_{t}\left(M_{\mathrm{GUT}}\right) \approx 0.5$. Eq. (1) means that the natural sparticle scale is $M_{1 / 2} \sim m_{0} \sim \mu \sim M_{Z}$ and that an accidental cancellation by a part in $\approx 100$ is needed if $M_{3}>1.1 \mathrm{TeV}$.

Eq. (1) can be used to fix the overall SUSY mass scale, such that the CMSSM model has two free adimensional parameters: the ratios $M_{1 / 2} / \mu$ and $m_{0} / \mu\left(\tan \beta=3\right.$ and $A_{0}=0$ are for the moment kept fixed). Such parameter space is plotted in fig. 1:

- The light-gray regions are theoretically excluded because the minimum of the potential is not the physical one: in the left region one would have $M_{Z}^{2}<0$ which means that the true minimum is at $v=0$; in the bottom-right region the potential is unstable when the two higgses have equal vev.

- The red region in the middle is theoretically allowed, but has now been experimentally excluded. The darker red shows the new region probed and excluded by LHC with respect to the previous LEP bounds, approximated to be $M_{2}>100 \mathrm{GeV}$.

- The green region is allowed. Indeed it is close to the boundary where $M_{Z}=0$ and thereby has $M_{Z} \ll m_{0}, M_{1 / 2}, \mu$. 

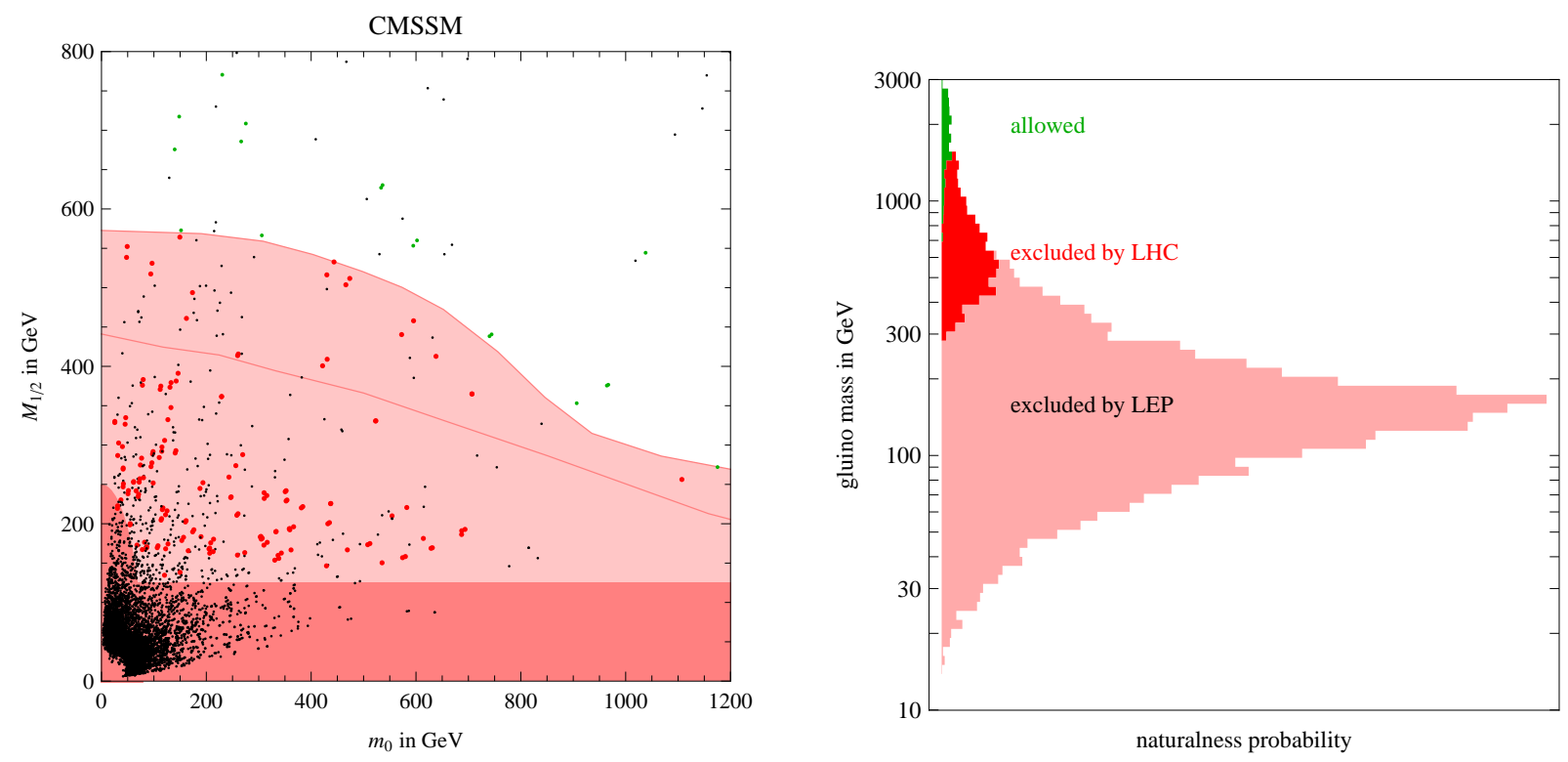

Figure 2: Left: naturalness scan of the CMSSM. Red points are excluded by LHC, black points have been excluded earlier, green points are allowed. The darker pink region was excluded by LEP and the pink region by early $L H C$ (the red lines show the various bounds from ATLAS and $C M S)$. Right: "naturalness probability distribution" for the gluino mass in the CMSSM. Only its tail was allowed after LEP, and the tail of the tail remains allowed after first LHC data.

The smallness of the allowed region is a manifestation of the "little hierarchy problem".

We now relax the restriction on $A_{0}$ and $\tan \beta$ (or equivalently $B_{0}$ ) and study naturalness proceeding along the lines of [1], as briefly summarized below.

We randomly scan the full theoretically allowed adimensional parameters of the model (the adimensional ratios between $m_{0}, M_{1 / 2}, \mu, A_{0}, B_{0}$ as well as the top Yukawa coupling $\lambda_{t}$, all renormalized at the unification scale) determining the overall SUSY mass scale and $\tan \beta$ from the potential minimization condition. Thanks to the last step, we sample the full CMSSM parameter space according to its natural density (rare accidental cancellations that make sparticles heavy happen rarely). We compute how rare are the still allowed sparticle spectra, as in [1] that claimed that only $5 \%$ of the CMSSM parameter space survived to LEP.

More precisely we perform the following scan

$$
m_{0}=\left(\frac{1}{3^{2}} \div 3\right)_{\log } m_{\mathrm{SUSY}}, \quad\left|\mu_{0}\right|, M_{1 / 2}=\left(\frac{1}{3} \div 3\right)_{\log } m_{\mathrm{SUSY}}, \quad A_{0}, B_{0}=(-3 \div 3)_{\operatorname{lin}} M_{1 / 2}
$$

and verify that it gives results similar to other possibilities such as

$$
m_{0},\left|\mu_{0}\right|, M_{1 / 2}=\left(\frac{1}{3} \div 3\right)_{\log } m_{\mathrm{SUSY}}, \quad A_{0}, B_{0}=(-3 \div 3)_{\operatorname{lin}} m_{0}
$$

or as

$$
m_{0},\left|\mu_{0}\right|, M_{1 / 2},\left|B_{0}\right|,\left|A_{0}\right|=(0 \div 1)_{\operatorname{lin}} m_{\mathrm{SUSY}} .
$$

where the pedices 'lin' and 'log' respectively denote a flat probability distribution in linear or logarithmic scale within the given range.

More formally, this is a Monte Carlo Bayesian technique that starts with an arbitrary non-informative prior probability density function (implicitly defined by the 'random scans' in 


\begin{tabular}{c|ccc}
$\begin{array}{c}\text { experimental } \\
\text { bound }\end{array}$ & \multicolumn{3}{|c}{ fraction of surviving CMSSM parameter space } \\
any $m_{h}$ & $m_{h}>100 \mathrm{GeV}$ & $m_{h}>110 \mathrm{GeV}$ \\
\hline LEP & $10 \%$ & $4 \%$ & $1 \%$ \\
LHC & $0.9 \%$ & $0.5 \%$ & $0.3 \%$
\end{tabular}

Table 1: Fraction of the CMSSM parameter space that survives to the various bounds.

eq.s (2) to (4)) and gives a set of points in parameter space with probability density roughly equal to the inverse of the various fine-tuning measures proposed to approximate the naturalness issue [6]. The above procedure makes no use of any fine-tuning parameter, and automatically takes into account all fine-tunings: not only the one needed to have $M_{Z} \ll m_{0}, M_{1 / 2}, \mu$, but also the one needed to have $\tan \beta \gg 1$, or the fine-tuning on $\lambda_{t}$ that can give a small or even negative $m_{0}^{2}$ coefficient in eq. (1), such that the $M_{3}^{2}$ term can be cancelled by $m_{0}^{2}$ rather than by $\mu^{2}$. The scanning is restricted to top quark masses within 3 standard deviations of the present measured value, $m_{t}=(173.1 \pm 1.1) \mathrm{GeV}[7]$.

A technical detail. The MSSM minimization equations generalize eq. (1) taking into account one loop corrections to the potential. To understand their relevance, we recall that at tree level the higgs mass is predicted to be $m_{h}^{\text {tree }} \leq M_{Z} \cos 2 \beta$, while at loop level it can be above the experimental limit $m_{h}>114 \mathrm{GeV}$. The effect of minimizing the one loop potential (rather than the tree level potential) is essentially equivalent to rescaling the overall SUSY mass scale by a factor $m_{h} / m_{h}^{\text {tree }}$, which helps naturalness.

We consider the three main bounds on sparticles, that can be roughly summarized as follows:

1) The LHC bound on (mainly) the gluino and squark masses is plotted in fig. $2 \mathrm{a} .{ }^{1}$ We find that this bound alone excludes about $99 \%$ of the CMSSM parameter space.

2) LEP tells that all charged sparticles (charginos, sleptons, stops...) are heavier than about $100 \mathrm{GeV}$, unless they are quasi-degenerate with the lightest supersymmetric particle. Such bounds alone excluded about $90 \%$ of the CMSSM parameter space [1].

3) The LEP bound on the Higgs mass $\left(m_{h}>114 \mathrm{GeV}\right.$ in the SM) is potentially even stronger but it is not robust and deserves a dedicated discussion.

As well known, the bound on the Higgs mass can exclude the whole MSSM, because the MSSM predicts at tree level a higgs lighter than $M_{Z}$ and at loop level a higgs lighter than about $125 \mathrm{GeV}$. The precise value logarithmically depends on the sparticle mass scale; we compute it using our own code and using the more precise SofTSusy code [8]. However, one can modify the MSSM to increase the predicted higgs mass (e.g. adding a singlet as in the NMSSM), avoiding the fine-tuning price of the higgs mass bound (and alleviating the whole fine tuning [9]). Furthermore, in regions of the MSSM parameter space with $\mu \sim M_{Z}$ and large $\tan \beta$ the higgs coupling to the $Z$ is reduced and a weaker bound $m_{h} \gtrsim 100 \mathrm{GeV}$ applies. More generically, the bound on the higgs mass can be weakened (down to about $100 \mathrm{GeV}$ ) modifying the theory such that the higgs has new dominant decay modes which are more difficult to

\footnotetext{
${ }^{1}$ The CMS and ATLAS collaborations computed bounds for $\tan \beta=3$ or 10 and $A_{0}=0[4,5]$. The dominant bound from events with jets and missing energy is essentially a bound on the gluino and squark masses, so that the dependence on $A_{0}$ and $\tan \beta$ can be neglected. The subleading bound from events with one lepton has only a moderate dependence on $\tan \beta$, that we also ignore.
} 


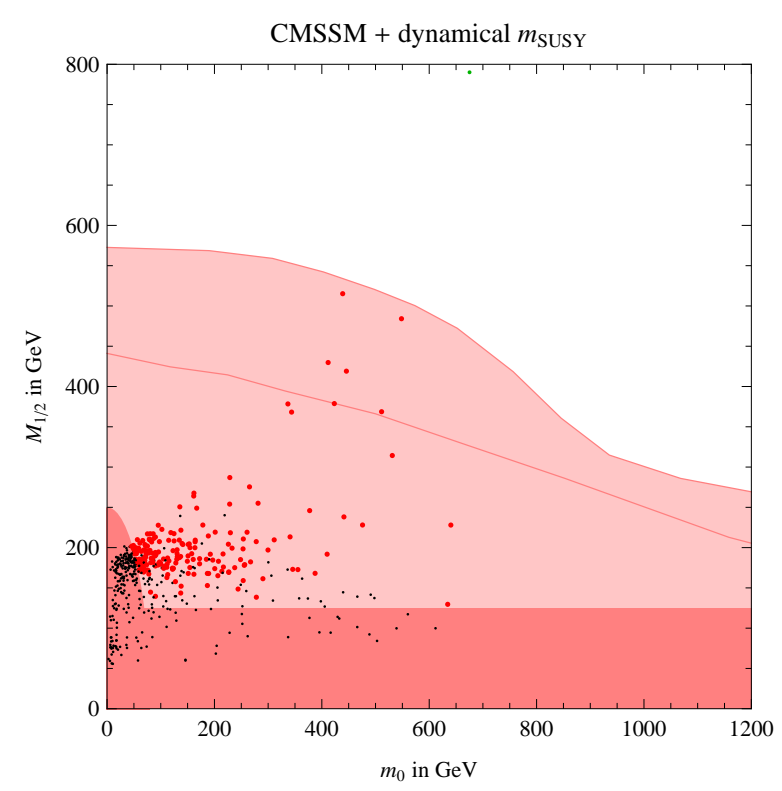

Figure 3: As in the previous figure 2a, but assuming that the sparticle mass scale is dynamically determined by minimizing the MSSM potential [13], such that it is naturally heavier than $M_{Z}$.

see experimentally, or which have not been experimentally studied [10]. Summarizing, many models can alleviate the fine-tuning problem related to the higgs mass.

The motivation for such models gets now washed out by the LHC bound, which has nothing to do with the higgs mass. Table 1 shows that naturalness remains a problem, even if one can circumvent the bound on the higgs mass.

In its last column we stick to the CMSSM and approximate the LEP bound $m_{h}>114 \mathrm{GeV}$ as $m_{h}^{\text {th }}>110 \mathrm{GeV}$, where $m_{h}^{\text {th }}$ is the higgs mass as computed by state-of-the-art codes [8], that have a theoretical uncertainty estimated to be about $\pm 3 \mathrm{GeV}$. With such a bound the allowed fraction of the CMSSM parameter space decreases down to about $0.3 \%$. The other scans (eq. (3) or eq. (4)) would give lower comparable fractions of allowed parameter space.

\section{Conclusions}

LHC data sharpen the "little hierarchy problem" of supersymmetry. Various tentative solutions were proposed. The simplest solution, a gluino lighter than what predicted by unification [11, 1], seems now excluded by the new LHC bound.

It is maybe useful to see the problem in this way: in SUSY models renormalization effects from the Planck scale down to the weak scale can radiatively break the weak symmetry, making the determinant of the squared higgs mass term negative below some scale $Q_{0}$ which in principle is anyway between the weak and Planck scales: the little hierarchy problem means that breaking of the weak gauge group happens at the last moment, $Q_{0} \sim v[2]$.

Therefore, one possible interpretation is that the Higgs is the pseudo-Goldstone boson of some symmetry broken at some scale $Q_{0}$ around the weak scale; however concrete models often look less plausible than the fine-tuning they avoid [12].

Another possibility considered in [13] is assuming that the overall scale of soft supersymmetry breaking is dynamically fixed by minimizing the weak part only of the potential (this assumption looks implausible, as recognized in [13]), such that the SUSY scale must be just 
below $Q_{0}$; the precise computation gives a neat prediction of the form $m_{\mathrm{SUSY}} \approx 4 \pi M_{Z} / 12$, where $4 \pi$ is a loop factor and the factor 12 counts the spins and colors in the most relevant diagram [13]. Applied to the CMSSM model such prediction is plotted as dashed line in fig. 1: it lies around the present allowed/excluded border. Fig. 3 shows the naturalness scan in the full parameter space: we see that even in this case LHC probed most of the parameter space. LEP tested SUSY masses around the $Z$ mass, and now LHC reached the next milestone, testing SUSY masses a loop factor above the $Z$ mass.

Maybe the weak scale is small due to anthropic selection, and attempts of keeping it technically small are like attempts of dragging the æther. The scenario of [13] was reconsidered in [14] with a different motivation: the authors imagine a supersymmetric multiverse where for some unknown reason the weak scale is "almost never" broken $\left(Q_{0} \ll m_{\text {SUSY }}\right)$, such that $Q_{0} \sim m_{\text {SUSY }}$ is "more likely". The qualitative expectation is similar to the prediction of [13], but $m_{\text {SUSY }}$ can be made heavier by order one factors making the "almost never" stronger, at the price of making MSSM vacua more rare than the SM with an unnaturally light higgs.

All these beautiful ideas and the history of the Michelson-Morley experiment teach us that a negative experimental search can have deep theoretical implications.

"History repeats itself, first as tragedy, second as farce". Karl Marx

Acknowledgements The author thanks Ben Allanach, Riccardo Rattazzi and Andrea Romanino for useful communications. This work was supported by the ESF grant MTT8 and by SF0690030s09 project.

\section{References}

[1] L. Giusti, A. Romanino, A. Strumia, Nucl. Phys. B550 (1999) 3 [arXiv:hep-ph/9811386]. See also A. Strumia, arXiv:hep-ph/9904247.

[2] R. Barbieri, A. Strumia, arXiv:hep-ph/0007265.

[3] WMAP collaboration, arXiv:1001.4744 and references therein.

[4] CMS collaboration, arXiv:1101.1628. ATLAS collaboration, arXiv:1102.5290. ATLAS collaboration, arXiv:1102.2357.

[5] LHC bounds on supersymmetry with more than $1 / \mathrm{fb}$ of integrated luminosity have been presented at the EPS-HEP conference on 25/7/2011 (web site http://eps-hep2011.eu) by G. Tonelli (for the CMS collaboration) and by D. Charlton (for the ATLAS collaboration), as well as in various parallel talks.

[6] R. Barbieri and G.F. Giudice, Nucl. Phys. B306 (1988) 63. B. de Carlos and J.A. Casas, Phys. Lett. B309 (1993) 320. G.W. Anderson and D.J. Castaño, Phys. Lett. B347 (1995) 300, Phys. Rev. D52 (1693) 1995. P. Ciafaloni and A. Strumia, Nucl. Phys. B494 (1997) 41 [arXiv:hepph/9611204]. P.H. Chankowski, J. Ellis and S. Pokorski, Phys. Lett. B423 (1998) 327 [arXiv:hepph/9712234]. P.H. Chankowski, J. Ellis, M. Olechowski and S. Pokorski, arXiv:hep-ph/9808275.

[7] Tevatron Electroweak Working Group and CDF and D0 Collaboration, arXiv:0903.2503.

[8] B.C. Allanach, Comput. Phys. Commun. 143 (2002) 305 [arXiv:hep-ph/0104145].

[9] R. Barbieri, L. J. Hall, Y. Nomura et al., Phys. Rev. D75, 035007 (2007) [hep-ph/0607332]. 
[10] For recent experimental effort, see ALEPH collaboration, JHEP 1005 (2010) 049 [arXiv:1003.0705].

[11] D. Wright, arXiv:hep-ph/9801449. G.L. Kane, S.F. King, Phys. Lett. B451, 113 (1999) [hep$\mathrm{ph} / 9810374]$.

[12] Z. Berezhiani, P. H. Chankowski, A. Falkowski et al., Phys. Rev. Lett. 96, 031801 (2006). [hep-ph/0509311]. C. Csaki, G. Marandella, Y. Shirman, A. Strumia, Phys. Rev. D73 (2006) 035006 [arXiv:hep-ph/0510294]. S. Chang, L. J. Hall, N. Weiner, Phys. Rev. D75, 035009 (2007). [hep-ph/0604076]. B. Bellazzini, S. Pokorski, V. S. Rychkov et al., JHEP 0811, 027 (2008). [arXiv:0805.2107]. B. Bellazzini, C. Csaki, A. Delgado et al., Phys. Rev. D79, 095003 (2009). [arXiv:0902.0015].

[13] R. Barbieri, A. Strumia, Phys. Lett. B490 (2000) 247 [arXiv:hep-ph/0005203].

[14] G. Giudice and R. Rattazzi, Nucl. Phys. B757 19 (2006) [hep-ph/0606105]. 